MATHEMATICS OF COMPUTATION

Volume 71, Number 240, Pages 1803-1806

S 0025-5718(02)01519-3

Article electronically published on May 28, 2002

\title{
CORRIGENDUM TO "PERIOD OF THE POWER GENERATOR AND SMALL VALUES OF CARMICHAEL'S FUNCTION"
}

\author{
JOHN B. FRIEDLANDER, CARL POMERANCE, AND IGOR E. SHPARLINSKI
}

We are indebted to Kelly Postelmans whose question drew our attention to a slip in the proof of Theorem 8 of [1. In particular, we asserted that for a fixed number $n$, the number of pairs of primes $p, l$ with $\operatorname{gcd}(p-1, l-1)<D$ and $\lambda(\lambda(p l))=n$ is at most $D \tau(n)$, an assertion which now seems unjustified. (The notation is defined below.) In this note we give a corrected proof of Theorem 8 .

As in [1] we consider the power generator

$$
u_{n} \equiv u_{n-1}^{e} \quad(\bmod m), \quad 0 \leq u_{n} \leq m-1, \quad n=1,2, \ldots,
$$

with the initial value $u_{0}=\vartheta$ (an integer coprime to $m$ ) and exponent $e$ (an integer at least 2). We recall that for an integer $n \geq 1$ the Carmichael function $\lambda(n)$ is the largest order occurring amongst elements of the unit group in the residue ring modulo $n$. As usual, $\varphi$ denotes Euler's function. We let $\tau(n)$ denote the number of natural divisors of $n$, we let $\omega(n)$ denote the number of divisors of $n$ that are prime, and we let $\Omega(n)$ denote the number of divisors of $n$ that are (either a prime or) a prime power. An integer $n$ is said to be squarefull if for each prime $p \mid n$ we have $p^{2} \mid n$. If $p^{a}$ is the largest power of the prime $p$ which divides $n$, and $a$ is at least 1 , we write $p^{a} \| n$. The letters $p, q, l$ always denote prime numbers.

The following is a slightly stronger form of Theorem 8 of [1].

Theorem 1. For $Q$ sufficiently large, for any $\Delta \geq 6(\log \log Q)^{3}$, and for all pairs $(p, l)$ of primes, $1<p<l \leq Q$, except at most $Q^{2} \exp \left(-0.1(\Delta \log \Delta)^{1 / 3}\right)$ of them, the following statement holds. For all pairs $(\vartheta, e)$ with

$$
1 \leq \vartheta \leq m-1, \quad 1 \leq e \leq \lambda(m), \quad \operatorname{gcd}(\vartheta, m)=\operatorname{gcd}(e, \lambda(m))=1,
$$

where $m=p l$, except at most $m \lambda(m) \exp (-\Delta / 4)$ of them, the period $t$ of the sequence $\left(u_{n}\right)$ given by (11) satisfies

$$
t \geq Q^{2} \exp (-\Delta)
$$

Proof. Let $\mathcal{S}$ be the set of pairs $(p, l)$ of primes with $1<p<l \leq Q$ and let $\mathcal{R}$ be the set of pairs $(p, l) \in \mathcal{S}$ for which all of the following hold:

(i) $\lambda(\lambda(p l)) \geq Q^{2} \exp (-\Delta / 3)$,

(ii) $\tau(p-1), \tau(l-1)<2^{\Delta^{2 / 5}}$ (so that $\omega(p-1), \omega(l-1)<\Delta^{2 / 5}$ ),

(iii) for each prime $q \mid \lambda(p l), \tau(q-1)<\exp \left(\Delta^{2 / 5}\right)$.

Received by the editor April 19, 2002.

2000 Mathematics Subject Classification. Primary 11B50, 11N56, 11T71; Secondary 11Y55, $94 \mathrm{~A} 60$. 
By Theorem 6 of [1] we have that the number of pairs $(p, l) \in \mathcal{S}$ that do not satisfy (i) is

$$
E_{1} \ll Q^{2} \exp \left(-0.11(\Delta \log \Delta)^{1 / 3}\right) .
$$

From the well-known bound

$$
\sum_{n \leq x} \tau^{2}(n) \ll x \log ^{3} x,
$$

it follows that the number of pairs $(p, l) \in \mathcal{S}$ that do not satisfy (ii) is

$$
E_{2} \ll Q^{2} \exp \left(-\Delta^{2 / 5}\right) \text {. }
$$

We now consider pairs $(p, l) \in \mathcal{S}$ that do not satisfy (iii). Suppose $\omega(q-1) \geq \Delta^{2 / 5}$ for some prime $q \mid \lambda(p l)$. We have

$$
\begin{aligned}
\sum_{n \leq Q, \omega(n) \geq \Delta^{2 / 5}} \frac{1}{n} & \leq \sum_{j \geq \Delta^{2 / 5}} \frac{1}{j !}\left(\sum_{q \leq Q}\left(\frac{1}{q}+\frac{1}{q^{2}}+\cdots\right)\right)^{j} \\
& =\sum_{j \geq \Delta^{2 / 5}} \frac{1}{j !}\left(\sum_{q \leq Q} \frac{1}{q-1}\right)^{j} \\
& \leq \sum_{j \geq \Delta^{2 / 5}} \frac{1}{j !}(c+\log \log Q)^{j}
\end{aligned}
$$

where $c$ is an absolute constant. Since $\Delta \geq 6(\log \log Q)^{3}$, it follows that if $Q$ is sufficiently large, the terms in the above series decay at least geometrically, so that

$$
\sum_{n \leq Q, \omega(n) \geq \Delta^{2 / 5}} \frac{1}{n} \leq \exp \left(-\frac{1}{3} \Delta^{2 / 5} \log \Delta\right) .
$$

Thus, the number of primes $p \leq Q$ such that $p-1$ is divisible by a prime $q$ with $\omega(q-1) \geq \Delta^{2 / 5}$ is at most

$$
\sum_{n \leq Q, \omega(n) \geq \Delta^{2 / 5}} \frac{Q}{n+1} \leq Q \exp \left(-\frac{1}{3} \Delta^{2 / 5} \log \Delta\right),
$$

as can be seen by forgetting just for the moment that $p$ and $q$ are primes and using only that they are integers at least 2. Now suppose that $q \mid p-1, \omega(q-1)<\Delta^{2 / 5}$, and $\tau(q-1) \geq \exp \left(\Delta^{2 / 5}\right)$. Note that if $\omega(n)<\Delta^{2 / 5}$ and $\tau(n) \geq \exp \left(\Delta^{2 / 5}\right)$, then $2^{\Omega(n)} \geq \exp \left(\Delta^{2 / 5}\right)>\exp (\omega(n))$, so that $\Omega(n)-\omega(n)>\frac{1}{3} \Delta^{2 / 5}$. (The constant $\frac{1}{3}$ can be improved to 0.75679 .. using the inequality $\tau(n) \leq(3 / 2)^{\Omega(n)-\omega(n)} 2^{\omega(n)}$.) Every such number $n$ may be factored as $n_{1} n_{2}$, where $n_{1}$ is squarefull, $\Omega\left(n_{1}\right)>\frac{1}{3} \Delta^{2 / 5}$, and $n_{2} \leq Q$. Thus the sum of reciprocals of such numbers $n \leq Q$ is at most

$$
\sum_{n_{1}} \frac{1}{n_{1}} \sum_{n_{2}} \frac{1}{n_{2}} \ll 2^{-\frac{1}{6} \Delta^{2 / 5}} \log Q \ll \exp \left(-\frac{1}{9} \Delta^{2 / 5}\right),
$$

where $n_{1}$ and $n_{2}$ independently run through integers of the above types. (Here we have used that the least squarefull number $n$ with $\Omega(n) \geq k>1$ is $2^{k}$ and that the sum of the reciprocals of the squarefull numbers that are at least $B$ is $\ll B^{-1 / 2}$, the latter following from partial summation and the fact that there are $O\left(x^{1 / 2}\right)$ squarefull numbers in $[1, x]$.) Thus, the number of primes $p \leq Q$ such that 
$p-1$ is divisible by a prime $q$ with $\tau(q-1) \geq \exp \left(\Delta^{2 / 5}\right)$ and $\omega(q-1) \leq \Delta^{2 / 5}$ is $\ll Q \exp \left(-\frac{1}{9} \Delta^{2 / 5}\right)$ and, by (2), this latter condition (on $\omega$ ) may be dropped. Hence, the number of pairs $(p, l) \in \mathcal{S}$ which do not satisfy (iii) is

$$
E_{3} \ll Q^{2} \exp \left(-\frac{1}{9} \Delta^{2 / 5}\right)
$$

We conclude that for sufficiently large $Q$,

$$
|\mathcal{R}| \geq\left(\begin{array}{c}
\pi(Q) \\
2
\end{array}\right)-E_{1}-E_{2}-E_{3} \geq\left(\begin{array}{c}
\pi(Q) \\
2
\end{array}\right)-Q^{2} \exp \left(-0.1(\Delta \log \Delta)^{1 / 3}\right) .
$$

We shall show that the conclusion of the theorem holds for every pair $(p, l)$ in $\mathcal{R}$. Let us fix some pair $(p, l) \in \mathcal{R}$ and put $m=p l$. We have, by (ii),

$$
\tau(\lambda(m)) \leq \tau(\varphi(m)) \leq \tau(p-1) \tau(l-1)<\exp \left(2 \Delta^{2 / 5}\right) .
$$

Further, using that $\varphi(a b) \mid \varphi(a) \varphi(b) b$ for all positive integers $a, b$,

$$
\tau(\lambda(\lambda(m))) \leq \tau(\varphi((p-1)(l-1))) \leq \tau(\varphi(p-1)) \tau(\varphi(l-1)) \tau(l-1) .
$$

Here, we have $\tau(l-1) \leq \exp \left(\Delta^{2 / 5}\right)$, by (ii). Moreover, for $(p, l) \in \mathcal{R}$, we have, by (ii) and (iii),

$$
\begin{aligned}
\tau(\varphi(p-1)) & \leq \prod_{q^{a} \| p-1} \tau\left(\varphi\left(q^{a}\right)\right)=\prod_{q^{a} \| p-1} a \tau(q-1) \leq \tau(p-1) \prod_{q \mid p-1} \tau(q-1) \\
& <\exp \left(\Delta^{2 / 5}\right) \exp \left(\Delta^{2 / 5} \omega(p-1)\right)<\exp \left(\Delta^{4 / 5}+\Delta^{2 / 5}\right)
\end{aligned}
$$

and the same bound holds for $\tau(\varphi(l-1))$. We conclude that

$$
\tau(\lambda(\lambda(m)))<\exp \left(2 \Delta^{4 / 5}+3 \Delta^{2 / 5}\right) .
$$

We apply Lemma 3 of [1] with $K_{1}=K_{2}=\exp (\Delta / 3)$. It follows that for sufficiently large $Q$, apart from at most

$$
\varphi(m) \varphi(\lambda(m))\left(\frac{\tau(\lambda(m))}{\exp (\Delta / 3)}+\frac{\tau(\lambda(\lambda(m)))}{\exp (\Delta / 3)}\right) \leq m \lambda(m) \exp (-\Delta / 4)
$$

exceptional pairs $(\vartheta, e)$, the period $t$ of the power generator satisfies

$$
t \geq \lambda(\lambda(m)) \exp (-2 \Delta / 3) \geq Q^{2} \exp (-\Delta),
$$

by (i), so the result follows.

We remark that Kelly Postelmans also pointed out to us a slip in the proof of Lemma 1 concerning reduced residues modulo a prime power $p^{m}$. This slip is easily fixed by replacing in the last paragraph of the proof our incorrect assertion that $g$ be a $d$-th power modulo $p^{m}$ by the condition that it be a $\lambda\left(p^{m}\right) / d$-th root of unity. (In case $p=2$, these are not quite the same.) 


\section{REFERENCES}

[1] J. B. Friedlander, C. Pomerance and I. E. Shparlinski, Period of the power generator and small values of Carmichael's function, Math. Comp., 70 (2001), 1591-1605.

Department of Mathematics, University of Toronto, Toronto, Ontario M5S 3G3, CANADA

E-mail address: frdlndr@math.toronto.edu

Department of Fundamental Mathematics, Bell labs, Murray Hill, New Jersey 07974-0636

E-mail address: carlp@research.bell-labs.com

Department of Computing, Macquarie University, Sydney, New South Wales 2109, Australia

E-mail address: igor@ics.mq.edu.au 